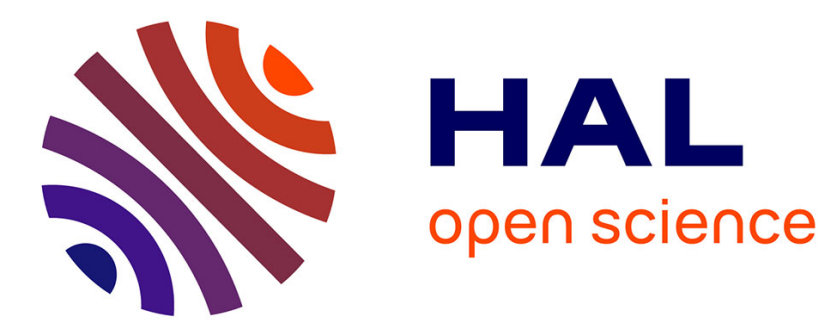

\title{
Des poussins au poivre, volailles aux tables épiques
}

Valérie Naudet

\section{To cite this version:}

Valérie Naudet. Des poussins au poivre, volailles aux tables épiques. Chantal Connochie-Bourgne. SENEFIANCE - Déduits d'oiseaux au Moyen Age, 54, PUP, pp.223-236, 2009, Déduits d'oiseaux au Moyen Age, 9782853997249. 10.4000/books.pup.4290 . hal-01506673

\section{HAL Id: hal-01506673 https://hal-amu.archives-ouvertes.fr/hal-01506673}

Submitted on 28 Apr 2017

HAL is a multi-disciplinary open access archive for the deposit and dissemination of scientific research documents, whether they are published or not. The documents may come from teaching and research institutions in France or abroad, or from public or private research centers.
L'archive ouverte pluridisciplinaire HAL, est destinée au dépôt et à la diffusion de documents scientifiques de niveau recherche, publiés ou non, émanant des établissements d'enseignement et de recherche français ou étrangers, des laboratoires publics ou privés. 


\section{9boOKS}
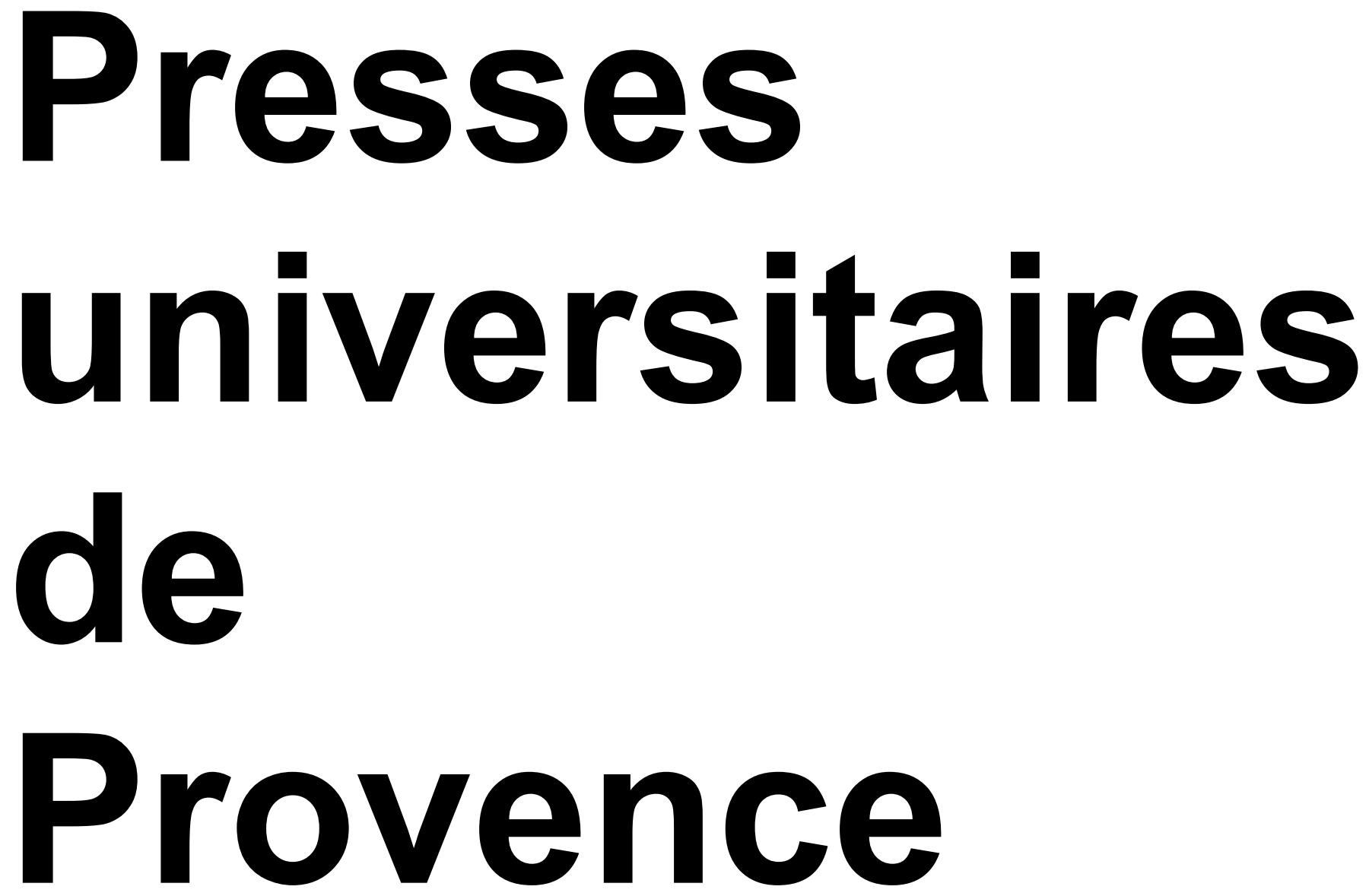

Déduits d'oiseaux au Moyen Âge

\section{Des poussins au \\ poivre, volailles \\ aux tables épiques}

Valérie Naudet 


\section{Texte intégral}

Tout le ciel cette nuit proclame

L'hécatombe des rossignols Aragon, Les Poètes

$1 \quad$ Lorsque le héros épique ${ }^{1}$ lève les yeux vers l'azur profond du zénith $^{2}$, les seules ailes qu'il y puisse voir sont celles des anges venus à sa rencontre ${ }^{3}$. De vol d'oiseaux, point. Lorsque le héros épique s'éveille, au matin de la bataille, c'est au son des messes chantées et au cliquetis des armes que l'on saisit. De chant d'oiseaux, point. Le fracas des armes et la fureur des hommes ne laissent que peu de place à la voix gracile du rossignol comme au vol amoureux de l'alouette ${ }^{4}$. Il en est en fait de l'oiseau comme de tout autre élément du décor : dans le monde épique étroitement centré sur le héros et la lutte qui l'obsède, les composantes de l'environnement ne sont mentionnées que lorsqu'elles présentent quelque rapport avec le personnage principal ou l'intrigue dans laquelle il est engagé. Dès lors s'expliquent aisément les (rares) apparitions des oiseaux dans les chansons de geste : si le héros n'a le temps ni de jouir de leur chant, ni de contempler leur vol, il peut en revanche leur trouver une certaine utilité. C'est ainsi que faucons, autours et aurions, fièrement juchés sur l'épaule ou le poing de leur maître, l'accompagnent durant ses parties de chasse, se laissent nourrir familièrement et en viennent à symboliser une chevalerie en paix avec les autres et elle-même ${ }^{5}$. On comprend alors la rareté de telles images. Cet usage de l'oiseau de proie, apanage exclusif de la noblesse, lui confère une valeur certaine. C'est pourquoi, cadeau de prix, il est souvent offert à quelqu'un que l'on cherche à honorer, apaiser ou amadouer. Il apparaît donc dans ces cortèges de présents où se mêlent animaux de chasse (chiens et oiseaux) qui disent l'espoir d'une paix à venir, raretés zoologiques et exotiques (chameaux, lions et ours et même dragons), épices, soieries et pierres précieuses 6 . 
Toutefois, ce n'est pas à l'épaule du chasseur ou dans la malle bien garnie de l'ambassadeur que l'on trouve les mentions les plus fréquentes des oiseaux dans les chansons de geste. C'est, paradoxalement peut-être, sur les tables, où, de prédateurs, ils sont devenus gibier pour enchanter les palais et satisfaire les solides appétits de nos héros. De fait, les quelques chansons de geste qui ont fait l'objet de notre dépouillement affichent du gibier à plume au menu avec une belle régularité et une fréquence qui interroge. Est-ce là un simple reflet d'une réalité alimentaire ? Mais alors de quelle époque ? $\mathrm{Ce} \mathrm{xII}^{\mathrm{e}}$ siècle qui a vu les poèmes épiques s'épanouir et se fixer à l'écrit, ou cette période carolingienne qui sert de cadre à nombre de fictions ? Ou bien ce goût pour les volatiles est-il une construction poétique ? Quelle fin sert-il alors ? Que disent de ceux qui les engloutissent les poussins au poivre et les paons rôtis?

Il arrive parfois qu'au soir d'une longue journée, de bataille ou de voyage, le trouvère lève le voile sur le temps de repos de ses personnages et nous donne à voir le repas qui leur est servi. Force est alors de constater que les tables sont souvent garnies d'oiseaux. Ainsi, lorsque Guillaume rentre à Barcelone, seul, après sa première défaite à Larchamp, Guibourc lui présente-t-elle un repas composé d'une épaule de sanglier à la broche, d'un pain de farine, de deux gâteaux, d'un grand paon rôti et d'un hanap de vin7. Le roi de Constantinople offre deux banquets à Charlemagne et à ses pairs lors de leur passage dans saville. À chaque fois,

Asez unt venesun de cerfs e de sengler

E unt grues e gauntes e poüns enpeverez

As pandant lur portent le vin e le clarez.

(v. 410-412 et $834-836)^{8}$

4 On remarque que Guillaume fait servir à Gillebert récemment échappé d'Orange le même menu et que luimême, déguisé, partagera avec le Sarrasin Arragon un repas tout à fait semblable, dans lequel le paon sera rôti et non cuisiné au poivre 9 . Après avoir incendié Origny, Raoul de Cambrai mangerait bien poons rostiz et bon cisnes pevreis ${ }^{10}$. 
Quant à Girart de Roussillon, réfugié dans Dijon, il se voit servir les désormais habituels poün et grues, que le poète rassemble sous le vocable générique de volatire quand il évoque le repas de Pierre de Mont-Rabei à Roussillon ${ }^{11}$.

La relative fixité des mets comme la répétition, parfois dans un ordre identique, des noms des oiseaux inviteraient volontiers à penser que ces vers sont une concession commode du trouvère à ce style formulaire si souvent évoqué à propos de la chanson de geste. D'autant que si la consommation de volaille est bien attestée par les historiens, elle ne saurait être semblable à celle que présentent les textes $^{12}$. En effet, les volatiles cités par les poètes n'appartiennent pas à la basse-cour d'élevage classique : paons, cygnes, grues, poussins et oies sauvages ressortissent à un autre registre et l'instante mention de leur présence à l'exclusive de tout autre oiseau, notamment domestique comme le poulet, peut difficilement être interprétée comme relevant d'un reflet plus ou moins fidèle à une certaine réalité historique, non plus celle de l'époque carolingienne que celle du XII ${ }^{\mathrm{e}}$ siècle capétien ${ }^{13}$.

6 Le régime alimentaire qui se met lentement en place dans l'Occident chrétien à partir du $\mathrm{Ix}^{\mathrm{e}}$ siècle est le fruit d'une acculturation réciproque entre deux modèles, le premier, gréco-romain, fondé sur le blé, l'huile d'olive et le vin, prônant un idéal de tempérance, le second, venant du nord de l'Europe, s'appuyant sur la trilogie viande, saindoux, cervoise et mettant à l'honneur les gros mangeurs ${ }^{14}$. Certes, nos chansons de geste, mêlant la viande avec le pain, servant du vin, offrent un état de fait qui résulte de l'interpénétration des deux modèles. Cependant, l'insistance des trouvères sur la partie carnée de l'alimentation est un indice de l'appartenance des mangeurs à la classe nobiliaire et chevaleresque. La viande, aliment onéreux, est devenue un indice de richesse, voire de luxe, que seul un petit groupe pouvait se permettre. L'abondance des plats de gibier à plumes sur les tables, effet de l'hyperbolisation épique, fonctionnait certainement comme un rêve d'idéal de satiété. La mention des oies sauvages ou des grues renforce cette 
connotation : ces animaux constituaient le fruit d'une chasse au vol, activité de plus en plus réglementée et réservée à la noblesse, au fur et à mesure de l'avancée dans le Moyen Âge. La noblesse du gibier signale celle du prédateur et du mangeur.

7 Quant aux paons et aux cygnes, s'ils ne sont pas forcément des proies du déduit d'oiseau, ils vont également dans le même sens d'une valorisation chevaleresque de celui qui les mange. Oiseaux d'ornement et non de boucherie, ils sont par leur inutilité même le luxe extrême, auquel leur insigne beauté venait, probablement, encore ajouter de la valeur ${ }^{15}$. Dès lors, le contexte dans lequel ils apparaissent, au soir même du massacre d'Origny ou de la bataille de Larchamp, est étrange. Il faut supposer que l'armée en campagne de Raoul transportait dans ses impedimenta ces luxueux volatiles ou bien voir en eux le fruit du pillage du bourg. Quant à la Chanson de Guillaume, si elle présente toujours la ville sur laquelle règne Guibourc comme un havre de paix dans une contrée hostile et en permanence ravagée par la guerre, le choix du paon comme plat servi à Guillaume paraît en contradiction avec la situation de la cité. Dans un cas comme dans l'autre, l'abondance gastronomique contraste violemment avec la situation des personnages. On a alors l'impression que ces poèmes concentrent dans ces passages l'essence de l'activité chevaleresque, métier des armes, volerie et luxe de vie, ce qui, dans le même temps, en dit long sur les espoirs de paix dans un monde où la chasse à l'oiseau est devenue un temps comme un autre de la guerre.

8 C'est donc comme un signe aristocratique que les trouvères présentent ces oiseaux dont ils garnissent si volontiers et si régulièrement la table de leurs personnages. Les animaux deviennent un symbole de domination autant que de luxe dont le noir versant serait la morgue et l'orgueil. L'ermite qui, au terme de leur fuite et d'une longue déchéance, recueille Girart et Berthe l'a bien compris. Dans sa pauvre masure enfouie au cour des ronces dans le plus profond d'une sombre vallée, il sert aux époux un pan d'orge pestrit a laisivers (v. 7567). On serait tenté de ne voir dans ce pain 
cendré et l'eau claire dont il est arrosé que la quotidienne pénitence d'un homme de Dieu retiré au désert, si le poète ne précisait pas que ces aliments sont offerts en lieu et place des usuels volatiles gastronomiques comme le pluvier :

Ne lor dona de[n]taz n'autres pluers

Mais pan d'orge [prestit] a laisivers

E l'aige freide e douce de fonteners.

(v. 7566-7568) $)^{16}$

Loin d'être un simple don, l'attitude de l'ermite est la pénitence donnée pour l'ancien mode de vie du héros. Les pluviers focalisent sur eux tous les excès dont Girart s'est rendu coupable : excès d'orgueil qui l'a dressé contre son roi jusqu'à le faire trébucher ; excès de gourmandise, peut-être, de celui qui se nourrissait de mets fins mais sans faim véritable. Le changement de régime alimentaire imposé par le saint homme n'est que le premier et le plus visible, à ce stade de la geste, des changements qui vont amener Girart, fermement guidé par Berthe, sur la voie de la rédemption ${ }^{17}$. Si le héros ne s'est pas damné à table, c'est à table néanmoins qu'il entamera sa pénitence.

10 Par ailleurs, outre cet aspect social, on peut discerner, dans le choix de mettre du gibier à la table épique, une autre composante, médico-sociale celle-là, comme s'il fallait, sous le couvert d'un discours scientifique, masquer les traces de ce genus aristocratique trahi par ce régime d'orgueil et de plume. En effet, dans l'univers médiéval tout entier ordonné autour de Dieu, s'instaure assez naturellement une hiérarchisation des êtres et des choses, et donc des aliments, en fonction de leur place dans le cosmos. On comprend alors que les oiseaux, êtres du ciel et de l'air, au plus proche de la sphère divine, fussent considérés comme les plus nobles des aliments. Dès lors, joue un principe d'analogie autant que d'adéquation entre le mangeur et le mangé : la nourriture la plus noble doit aller dans les estomacs les plus nobles comme leur convenant le mieux. Nos héros sont donc nourris selon leur nature même ${ }^{18}$. On mesure alors combien est grand le fossé entre les pluviers auxquels était habitué le 
couple comtal du Girart de Roussillon et le pain d'orge offert par l'ermite. Ce pain est doublement noir, de cette lourde noirceur de la terre dont il est issu, contrastant avec le caractère aérien et coloré (songeons à la blancheur du cygne ou au chatoiement du paon) des oiseaux : il n'est pas pétri de blé, qui donne un pain blanc, prisé de la noblesse, mais d'orge, céréale noire des pauvres à cause de son rendement plus élevé que celui des autres végétaux ${ }^{19}$. La couleur foncée de la céréale n'est pas ici fortuite : elle emblématise cette terre dont Girart doit apprendre à extraire le sang noir, ce charbon qui va assurer sa subsistance, maintenant qu'il est déchu des hautes sphères de la société chevaleresque. En outre, à cette orge est mêlée de la cendre, froid et mort résidu de la flamme, dans lequel on pourrait lire les restes de cette colère, humeur liée analogiquement au feu, qui consuma le héros dans sa lutte mortifère contre le roi.

11 De plus, pour reprendre une expression de Massimo Montarani, la viande, c'est " la viande des forts ${ }^{20}$. Et ce, à double titre dans nos poèmes : apanage d'un petit groupe social seul capable de s'offrir ce mets onéreux, elle est aussi symboliquement l'aliment qui, apportant du sang et de la chair, vient nourrir la force du guerrier. L'insistance sur la sauvagine ajoute une notation particulière : c'est la férocité inhérente au monde extérieur que vainc le héros à chaque fois qu'il mange un animal sauvage, tout autant qu'il s'en approprie les qualités par le jeu de la digestion. Dans la complexe chimie du corps, la dangerosité du gibier aide à alimenter celle qui doit permettre au combattant de s'affirmer sur le champ de bataille et c'est en partie l'air de la forêt qui s'exhale dans le furor du guerrier. Si nos oiseaux sont peu aptes à incarner cette férocité et cette dangerosité qui semblent être davantage les qualités du grand gibier, il n'en reste pas moins que, comparés à une poule ou un poulet, ils apportent une charge supplémentaire dans leur connotation, véhiculant sous leurs ailes un peu de ces grands espaces et de ce monde sauvage parce qu'inconnu qu'eux seuls sont en mesure de parcourir. C'est comme si, en 
ingérant de l'oie ou de la grue à la table impériale de Constantinople, les douze pairs maîtrisaient un peu mieux la vastitude de la Création, ce que confirme par ailleurs la chanson qui raconte le pèlerinage de Charles. Les pas de nos pèlerins les ont guidés dans un tour de la Méditerranée, depuis l'Occident et Saint-Denis, jusqu'à Jérusalem, puis Constantinople, mais aussi dans une maîtrise symbolique de ce monde ${ }^{21}$, puisque chacune des villes incarnant une fonction dumézilienne, la primauté reconnue de Charles et des siens partout où ils passent indique une souveraineté universelle de l'empereur franc.

12 Pour en finir avec la diététique, il faut examiner la façon dont sont accommodées nos viandes. Deux recettes principalement figurent dans les poèmes, sans qu'il soit parfois possible de déterminer si elles sont exclusives l'une de l'autre. Les oiseaux sont rôtis et/ou au poivre ${ }^{22}$. La cuisson à la broche comme l'assaisonnement au poivre, épice qui assèche et échauffe, ont la propriété de pouvoir contrebalancer le caractère humide des oiseaux, créatures influencées par leur milieu, l'air, élément par nature chaud et humide ${ }^{23}$. La dessiccation par le feu ou l'épice fait des grues et autres paons un plat parfaitement équilibré auquel il ne reste plus que la propriété calorifique, idéale pour entretenir la nécessaire et salutaire bile jaune ou colère, source de l'agressivité que ces hommes doivent déployer sur le champ de bataille. On peut alors tenter d'expliquer la différence que le poète du Guillaume introduit entre le repas de Girart au soir de la mort de Vivien et celui de Guillaume quatre jours plus tard ${ }^{24}$. Girart avale une épaule de sanglier, un grant pain a tamis (v. 1047) et un grant mazelin de vin (v. 1048), soit le même repas que Guillaume à l'exception de deux gâteaux et du paon rôti. Or si l'on considère que l'apport principal des gâteaux est probablement constitué de farine, également présente dans le pain, et d'œufs, aliment à relier à nos oiseaux, seuls ces derniers sont en balance entre les deux repas. Pourquoi sont-ils servis à l'oncle et non au neveu ? C'est essentiellement une différence d'âge qui apparaît entre les deux guerriers. Or la théorie humorale ${ }^{25}$ 
fait évoluer le tempérament de l'homme en fonction de ce paramètre : la jeunesse est en général considérée comme sanguine, c'est-à-dire chaude et humide ; quant à la maturité, elle est placée sous le signe de la bile jaune ou colère, chaude et sèche. Le paon, desséché par son mode de cuisson, contrarierait la nature sanguine de Girart. Quant à l'homme mûr qu'est Guillaume, il a besoin de reconstituer sa chaleur interne, opération que les oiseaux et les œufs vont l'aider à accomplir. Il est certes difficile de faire de l'un de nos plus vieilles chansons de geste et de surcroît l'une de celles à la transmission la plus problématique un traité de diététique à l'usage du guerrier. Nous croyons, toutefois, qu'il est possible d'entrevoir dans les minces interstices laissés par le jeu de la répétition formulaire une ouverture vers un savoir d'ordre scientifique.

13 Finalement, les paons, grues, oies, gantes, cygnes, pluviers et autres volatiles garnissant d'abondance les tables épiques répondent à une certaine logique : ces oiseaux sont avant tout un signe extérieur d'appartenance de celui auquel ils sont servis à la classe chevaleresque, noble et guerrière. Cependant, nous avons cru déceler dans ce signe autre chose, de plus profond, comme une adéquation et une congruence entre le mangeur et le mangé, la nature de l'oiseau et son mode de cuisson étant parfaitement adaptés à celle du héros.

14 Toutefois, on ne se contente pas de manger, on parle également de manger! Deux scènes prennent alors un relief particulier en ce qu'elles envisagent par le biais du langage un usage déviant, voire dévoyé, de nos oiseaux.

15 La Chanson de Guillaume offre la première de ces scènes dans le cadre palatin et parisien de la cour royale ${ }^{26}$. La reine, en s'immisçant dans l'échange entre Louis et Guillaume pour empêcher le seigneur de porter secours au vassal en danger, attire contre elle la vindicte de son frère qui explose en une longue et insultante tirade, l'accusant d'ivresse (v. 2599 et 2616), de parler à tort et à travers en disant du mal des gens (pudneise surparlere, v. 2603 et 2611), de se vautrer dans un luxe inouï au coin de ronflantes cheminées 
(v. 2614) sous de moelleuses couvertures (v. 2617), de se livrer à la fornication, accueillant dans sa couche tout ce que le royaume compte de lâches débauchés et de prêtres lubriques (v. 2604-2610 et 2618-2619) et puis... de manger des pudcins en pevrees (v. 2615). Dérisoire, cette dernière accusation dans le flot de violence verbale qui l'entoure ? Certes non, les poussins au poivre s'intègrent de manière cohérente dans la liste des reproches que l'on peut classer en trois catégories : la gourmandise, la luxure et un péché qui ne fait pas partie des sept capitaux, mais qui demeure l'une des préoccupations majeures des théologiens médiévaux, le péché de langue ${ }^{27}$. En outre, la façon dont le personnage s'adonne à ces pratiques peccamineuses la rend doublement coupable puisque l'on y décèle, à travers les paroles de Guillaume, un plaisir évident ainsi qu'un abandon sans frein. C'est bien l'excès, de bouche, de sensualité, de propos qui résume l'attitude de la reine et la conduit à un crime bien plus grave aux yeux de Guillaume, l'homme qui usa sa jovente ${ }^{28}$ belle à l'inlassable service de son roi : la trahison. Selon le héros, la trahison de la souveraine est à multiples facettes : elle trahit son statut de reine en s'acoquinant avec la lie de la société ; elle trahit son époux et les vœux de mariage qu'elle a prononcés devant Dieu ; elle trahit enfin sa nature de femme par son régime alimentaire aux antipodes de ce qu'elle devrait pratiquer. En effet, par nature, la femme est froide et humide ${ }^{29}$; or les aliments dont elle abuse, le vin comme les poussins au poivre, visent à provoquer sécheresse et surtout chaleur. Cette chaleur interne, née de la coction de la digestion, vient s'ajouter à celle dispensée par les cheminées crépitantes et les couettes douillettes pour nier complètement ce qu'est, essentiellement, une femme. Dès lors, il n'est pas impossible de penser que ses débordements luxurieux puissent être l'effet de l'excès calorifique dénaturant le personnage.

16 Par ailleurs, il convient de remarquer l'absence de nom de volatile, qui laisse ainsi, seul pour fournir l'apport sémantique, le terme de pudcin. Or celui-ci, désignant le petit, exhibe la gourmandise sans frein de la reine. On peut, 
en effet, se demander dans quelle mesure, l'oiseau, sous une forme aussi petite, est apte à rassasier. Le met, délicat et recherché, relève plus de la gourmandise, et donc du péché, que de la simple et utilitaire alimentation. La tension est alors extrême dans le poème entre cet état de la table curiale, garnie à foison de plats de luxe pour les plaisirs de bouche d'une seule, et le champ de bataille, sur lequel les hommes, en nombre, sont à la peine et à la faim ${ }^{30}$. C'est le scandale de ce contraste que dénonce Guillaume dans le heurt brutal de ces deux images : celle d'un Vivien, dont le nom est justement rappelé quelques vers avant celui des poussins (v. 2607), mourant de soif, condamné à souiller son corps de l'eau saumâtre, croupie et rougie du sang et de la chair des combattants d'un pauvre ruisseau sous l'ardent soleil de Larchamp et celle d'une femme, tout occupée d'ellemême et de ses plaisirs, s'adonnant à tous les péchés du corps dans l'atmosphère feutrée de la cour ${ }^{31}$.

17 Au final, les poussins au poivre sonnent dans la tirade de Guillaume aussi violemment et brutalement que les autres invectives. Ces oisillons, en apparence anodins, sont gros d'accusations de trahison, de nature.

18 Dans une autre chanson, dans la bouche d'un autre héros, d'autres noms d'oiseaux sont invoqués, tout aussi porteurs de funestes présages. C'est à Origny, au soir du sac du bourg et du meurtre de Marsent, que Raoul de Cambrai, mis en appétit par une journée de violents efforts et l'excitation du combat, réclame à son sénéchal :

« Del mangier pense, si feras grant bonté :

poons rostiz et bons cisnes pevreis

et venoison a molt riche plenté, qe tout li pires an ait tot a son gré. » (v. 1382-1384)

19 Tout se passe comme si le sang versé dans les rues du bourg appelait du sang nouveau, celui charrié par les pièces de gibier réclamées d'abondance. Quant aux paons rôtis et aux cygnes au poivre, c'est probablement pour leur pouvoir calorifique qu'ils sont demandés : là encore, la chaleur de la 
bataille réclame un prolongement, si ce n'est un substitut. L'impression est donnée que Raoul le desreé ${ }^{32}$ recherche à table les mêmes sensations fortes que sur le champ de bataille. Mais dans cet échauffement excessif qui lui brouille les sens, le personnage a oublié l'essentiel : le temps du carême et du jeûne, le temps de l'interdiction de toute viande. Le poisson, aliment emblématique de cette période de restriction, vivant dans l'eau, est l'antithèse absolue de l'oiseau aérien. En voulant se nourrir selon sa nature de colérique, Raoul contrevient à l'usage social et fait fi d'un tabou religieux. La réaction du sénéchal est, à cet égard, sans ambiguïté : après un triple signe de croix, il ose contredire son seigneur, ne lui laissant aucun doute sur le fond de ses pensées. Ce qui est intéressant alors, c'est l'ordre dans lequel il adresse ses reproches à Raoul :
« Nomeni dame! que avez empensé ?
Vos renoiés Sainte crestienté
et baptestire et Dieu de maïsté !
Il est caresme qe on doit jeüner -
li grans devenres de la solempnité
qe pecheor ont la crois aouré.
Et nos chaitif qe ci avons erré,
les nonnai[n]s arces, le mostier violé,
ja n'en serons envers Dieu acordé
se sa pitiés ne vaint no cruauté. »
(v. 1389-1398)

20 C'est tout d'abord le constat épouvanté d'un triple sacrilège, contre la chrétienté, le baptême et la majesté divine ; puis l'explicitation de la cause : non seulement on est en plein carême mais en plus le vendredi saint, ce qui implique en premier lieu le jeûne, dont le caractère exprès est rappelé par le verbe devoir (v. 1392). Ce n'est que dans un second temps, et comme un ajout venant inutilement alourdir un faix déjà trop lourd (la conjonction $e t$, à l'ouverture du vers 1395, contribue à créer cet effet d'accumulation), que les horreurs commises à Origny sont évoquées, sorte de facteur aggravant d'un cas autrement désespéré. On remarquera, ultime et horrifique détail, que les religieuses subissent in 
fine le même sort que les oiseaux réclamés par Raoul, desséchées et brûlées à la flamme de la colère du desreé.

21 Par ailleurs, cette scène est préparée par une autre, en amont de l'épisode, qui voit, déjà, Raoul parler inconsidérément. Une fois encore, des oiseaux apparaissent dans son discours, lorsqu'il donne ses ordres pour établir son camp dans l'abbaye :
«Mon tré tendez em mi liu del mostier
et en ces porches esseront mi sonmier ;
dedens les creutes conreés mon mangier ;
sor les crois d'or seront mi esprevier ;
devant l'autel faites aparillier
un riche lit ou me volrai couchier ;
au crucefis me volrai apuier
et les nonnains prendront mi esquier. »
(v. 1058-1065)

22 Les oiseaux ne sont pas de ceux que l'on mange, mais de ceux qui chassent, ce qui est normal puisque la curée que constitue le massacre des religieuses n'a pas encore eu lieu. Du coup le blasphème ne porte pas sur l'interdit alimentaire du carême, mais est constitué de la profanation d'un lieu saint et de l'orgueilleuse volonté du personnage de se substituer au Christ, dans une reconstitution dégradée et avilissante de la scène de la crucifixion ${ }^{33}$, dont les croix destinées à servir de perchoir de luxe aux oiseaux de proie sont un élément marquant.

23 L'incendie d'Origny est donc encadré de deux prises de parole, autoritaires et impérieuses, de Raoul, qui, toutes deux blasphématoires, envisagent un oiseau comme support d'un sacrilège. Pourtant, bien que proférées, d'une certaine manière, ces paroles vont rester lettres mortes : Raoul accepte dans les deux cas d'entendre les conseils avisés de son entourage ${ }^{34}$ et au bout du compte, les éperviers ne nicheront pas sur les crucifix non plus que paons et cygnes ne garniront sa table le vendredi saint. Pour autant il est difficile de leur dénier toute efficace et le personnage va bien passer de la parole à l'acte avec l'incendie du monastère.

24 Dans le monde désorbité qu'est celui de Raoul de Cambrai 
plus que dans tout autre, les oiseaux servis à table, auquel on peut associer les animaux de volerie, sont la matérialisation du genus aristocratique. Et à l'orgueil démesuré du personnage vont correspondre des volatiles porteurs de blasphème. À chaque fois qu'un nom d'oiseau apparaît dans la bouche du héros, un sacrilège y est attaché. C'est peut-être pour cela que le ciel de cette chanson reste vide et silencieux, non seulement de toute créature ailée mais également de toute manifestation divine, comme s'il était impossible à Raoul d'aspirer à s'y élever. Aussi est-ce vers la terre, noire et froide, que le personnage retombe, après avoir adressé une dernière prière à la douce dame del ciel (v. 2952). On sait que le trouvère ne se prononce pas sur l'efficace de ses ultimes paroles : est-il possible qu'après avoir fait monter vers les cieux la fumée nauséabonde du sacrifice dévoyé d'Origny, on y puisse chercher du secours?

25 Mais peut-être le poème de Raoul de Cambrai ne constituet-il pas le pire de ce qui peut se faire, dans la chanson de geste, en termes de gibier à plume. En effet, si les oiseaux sont convoqués par la parole blasphématoire, les gestes envisagés n'ont pas lieu et c'est un autre crime qui est commis. En revanche, le trouvère de Garin le Loheren franchit un pas supplémentaire dans la violence liée à la table et aux oiseaux.

26 Dans l'interminable conflit qui ensanglante sur plusieurs générations les relations entre Lorrains et Bordelais, le mariage de l'empereur Pépin avec Blanchefleur, l'héritière de Maurienne, aurait pu constituer une pause. Mais lors du banquet nuptial, une querelle éclate au sujet du service du roi et, en quelques instants, la rixe devient générale. Begon de Belin, dressant alors un plat en cuisine, pour voler au secours de son frère Garin, s'arme du premier objet qu'il trouve, un hastier [...] plain d'oiselés qui sunt chaut et rosti (v. 6118-6119) $)^{35}$ et entraîne après lui une troupe de marmitons. La broche s'avère une arme efficace entre les mains expertes du héros ; bonne est la broche de fer apointeïs (v. 6120) précise même le poète sans que l'on puisse déterminer avec exactitude l'usage, culinaire ou 
belliqueux qui vaut à l'objet cette appréciation flatteuse. Les vers suivants vont toutefois lever l'ambiguité, Begon blessant successivement Ysoré de Boulogne et Guillaume de Monclin, le texte renonçant à compter ensuite pour laisser la place à l'hypotypose classique qui donc veïst (v. 6132-6134 reprise v. 6144-6146 ${ }^{36}$ ), qui signale un temps fort du combat. Ce marqueur stylistique est bien l'indice que la broche et ses oiseaux rôtis sont considérés comme une arme à part entière qui équipe superbement un magnifique guerrier, comme en témoignent les qualificatifs qui le flanquent : le palasin (v. 6132) et le preu conte hardi (v. 6144). Par deux fois, le trouvère appelle l'attention du public sur le seigneur de Belin donnant à admirer les coups du héros dans un combat tout aussi digne qu'un autre. Mais, dans le même temps, se dessine en creux un monde que la violence et la guerre ont complètement gangrené, incapable de déguster quelques oiseaux rôtis. À la table nuptiale du couple impérial, il était prévu de servir les oiselés avec faste comme il se doit d'un mets de luxe symbolisant la fête et surtout, dans le contexte d'une noce, se chargeant de toutes les valeurs positives du banquet unissant les convives dans une concorde retrouvée. Mais tout est fait pour entraver ce processus : l'hymen célébré n'est pas celui que l'on attendait, les invités s'envient au point de se battre à table et le repas n'a même pas le temps de finir de cuire, puisque c'est encore embrochée que la volaille fait son apparition dans la salle du banquet. Il n'y a plus d'occasions de se rassembler ${ }^{37}$, de se réjouir comme il n'y a plus d'objets anodins, tout étant susceptible d'être dévoyé.

27 Comme dans Raoul de Cambrai, cette scène a un antécédent : une broche à rôtir a déjà fait l'objet d'un tel détournement. Dans la salle palatine de Monloon, lors du compte rendu à Pépin de l'expédition de Maurienne qui a vu se fissurer pour la première fois l'amitié unissant Lorrains et Bordelais, Garin perd son calme devant l'évidente mauvaise foi de Fromont et, première apparition du motif, la querelle s'envenime au point que l'on en arrive aux mains. Les adversaires étant désarmés comme le réclamait la situation, 
ils se voient contraints de s'équiper au mieux : Garin, avisant un écuyer qui passait muni d'un hastier (v. 2359), s'en empare. Mais le tableau est à peine esquissé, simple ébauche de ce que sera celui des noces de Pépin. Tout d'abord, si le mot est identique, hastier, on remarque que, dans cette première occurrence, rien n'est fait pour le mettre en valeur : pas de précision quant à son emploi, pas de mention de volaille rôtie ou à rôtir. Par ailleurs, il n'est pas question de montrer Garin dans sa gloire de chevalier combattant par le jeu d'une hypotypose qui fonctionne un peu comme un ralenti sur image. Il est vu se défendant contre un coup d'Hardrés, puis le relais est pris par son neveu dont l'arrivée providentielle à la cour royale va faire basculer la mêlée en faveur des Lorrains. Bref, tout est fait pour éloigner le Lorrain de l'univers des cuisines, comme s'il fallait éviter que ne s'imprimât dans la rétine du public l'image de ce guerrier armé d'une broche à volaille. L'examen du ms $F$ confirme cette impression puisque l'on y voit Garin s'armant d'un échiquier (v. 2209) et non d'un ustensile de cuisine. C'est que les frères lorrains incarnent deux facettes de la fonction guerrière ${ }^{38}$ : à Begon le penchant vers la force brute et donc l'univers de la cuisine et le combat avec une rôtissoire ; à Garin l'aspiration à un art du combat plus proche de l'escrime. On comprend alors qu'il n'eût pas été judicieux de brouiller cette perception dès l'aube de la geste. Mais, si la pique à volaille ne saurait être l'arme emblématique de l'aîné des Lorrains, elle convient, en revanche, parfaitement à son cadet, lui qui, se servant de son épée comme d'un couteau à découper, éventrera son adversaire de duel pour lui arracher le cœur et le jeter au public, lui, enfin, qui, chassant, verra la situation se retourner contre lui et sera abattu comme un vulgaire gibier. 28 Ainsi la broche et ses oiselés disent l'adéquation profonde entre le personnage de Begon de Belin, guerrier de force brute, et les puissances de la nature. Il est le seul capable de les apprivoiser, un temps, pour les mettre au service de la cause lorraine. Mais, ce faisant, il met au jour l'importance de la faille qui s'est ouverte dans l'univers dessiné par le 
poète : dans ce monde en pleine déliquescence, la sauvagerie et la bestialité ont droit de cité dans une salle palatine où l'on célèbre les noces de l'empereur.

29 Pour finir, il faut redire que l'oiseau ne constitue pas un motif épique majeur. Pour autant nous avons cru pouvoir déceler, à l'examen des tables dressées par les trouvères, dans la fréquence et la régularité des plats de gibier à plume, une intention qui ne tenait pas uniquement de la formule descriptive. Animal de venaison, donc noble, créature ailée au plus proche du Créateur dans la géographie symbolique du monde, être d'air, l'oiseau rôti ou au poivre est l'aliment qui semble convenir le mieux à la nature du chevalier. Et c'est l'adéquation entre mangeur et mangé qui permet au poète du Guillaume ou du Raoul, par exemple, de jouer sur une gamme d'effets obtenus en changeant les variables : le mangeur n'est pas un chevalier mais une femme qui voit sa nature contrariée par son régime alimentaire ; le mangeur est un chevalier dont l'orgueil démesuré va jusqu'à influencer tout ce qu'il touche, y compris la nourriture, muant cygnes et paons, mais aussi ses éperviers de chasse, en des objets de sacrilège.

30 Ainsi l'oiseau n'intéresse-t-il ni pour son ramage ni pour son plumage. Seule sa chair semble vraiment prisée dans les chansons de geste de notre corpus. Toutefois, dans l'univers violent et agité de ces poèmes épiques, les temps de fête et de banquet, de partage gastronomique et convivial sont rares : l'oiseau servi participe davantage à la reconstitution de forces perdues dans le combat qu'à une dégustation gourmande, quand il n'est pas, tout simplement, transformé en arme mortelle. On peut alors se demander si l'amertume n'est pas la saveur dominante de cette chair : amertume de ce mets que Raoul réclame en blasphémant, que Girart mange quand Charles tient son fief héréditaire de Roussillon ou que Guillaume avale en solitaire au soir de sa défaite.

\section{Notes}

1. Servent de support à cette étude La Chanson de Roland, éd. I. Short, Turnhout, Brepols Publishers, t. I, 2005 ; La Chanson de Guillaume, 
éd. Fr. Suard, Paris, Bordas, Classiques Garnier, 1991 ; Le Voyage de Charlemagne à Jérusalem et à Constantinople, éd. P. Aebischer, Paris, Champion, CFMA 115, 1965 ; La Chanson de Girart de Roussillon, éd. W. M. Hackett, trad. M. de Combarieu du Grès et G. Gouiran, Paris, Lettres Gothiques 4534, 1993 (pour cette chanson à la langue si difficile, nous donnons en note la traduction qui se trouve en regard du texte); La Prise d'Orange, éd. Cl. Régnier, Paris, Klincksieck, $1986\left(7^{\mathrm{e}}\right.$ édition) ; Garin le Loheren, according to Manuscript A (Ars 2983), éd. J. E. Vallerie, Columbia, 1947 et Garin le Loherenc, éd. A. IkerGittleman, Paris, Champion, CFMA 117, 118, 119, 1996-1997 pour le ms $F$ et la varia lectio et Raoul de Cambrai, éd. S. Kay, trad. W. Kibler, Paris, Lettres Gothiques 4537, 1996.

2. Sur le temps qu'il fait dans les chansons de geste, on se reportera aux études d'A. Labbé, "Ciel météorologique et Ciel mystique dans les chansons de geste ", Observer, lire, écrire le ciel au Moyen Âge. Actes du colloque d'Orléans, avril 1989, éd. B. Ribémont, Paris, 1991, p. 127152 et « Le Ciel de Renaut de Montauban : climat, intempéries, signes divins », Entre Épopée et légende : les quatre fils Aymon ou Renaut de Montauban, Actes du colloque de Reims-Charleville, octobre 1995, études publiées sous la direction de D. Quéruel, Langres, D. Guéniot, t. II, p. 9-42, en particulier la note 1 pour une bibliographie plus abondante.

3. La plus célèbre des cohortes angéliques du ciel épique est sans contexte celle qui vient escorter l'âme de Roland jusqu'aux portes du paradis (Roland, v. 2389-2396), mais l'on songera également à celle de Renaut de Montauban, v. 14223, éd. J. Thomas, Genève, Droz, 1989 ou à celle de saint Loup de Troyes mort en défendant sa ville dans le prologue de Garin le Loherenc, éd. A. Iker-Gittleman, v. 673. Voir A. Labbé, "Ciel météorologique... », op. cit. Notons toutefois que dans la plupart des cas étudiés, le ciel reste vide. On appréciera d'autant plus l'étrange vol d'oiseaux en tous genres qui fond sur Naymes au sommet d'Aspremont (La Chanson d'Aspremont, éd. L. Brandin, Paris, Champion, CFMA 19, 1970, v. 1967-1986.)

4. Les chansons de geste de cette étude présentent presque toutes la particularité de ne pas faire entendre le chant des oiseaux dans les reverdies qui les émaillent. Dès lors, les exceptions prennent un relief qui mérite attention : ce sont la Prise d'Orange et Raoul de Cambrai qui se distinguent, textes que leur date probable isole du groupe des poèmes les plus anciens. Dans les deux cas, les pépiements égayant les strophes vernales ne sont pas des formules toutes faites. Dans la geste du Nord, c'est au lendemain de son mariage et surtout de son enlèvement que Béatrix entend, depuis sa prison, le chant des oisillons. Le début printanier, longuement développé (v. 6033-6044), souligne 
d'une noire ironie tout ce dont aurait dû profiter l'héroïne, fraîche épousée de l'homme qu'elle aime que la rancune tenace d'un monarque autoritaire lui a ôté. Quant à la Prise d'Orange, ce sont les strophes III et IV qui présentent Guillaume appuyé à la fenêtre de son palais de Nîmes. Là encore le jeu d'intertextualité avec la lyrique courtoise est notable : l'exubérance de la nature rend d'autant plus criant le vide de l'existence du héros et de ses proches. En effet Nîmes est une place forte conquise récemment et la cour, avec dames et pucelles, n'a pas encore suivi. Les hommes en sont réduits à un mâle compagnonnage que l'arrivée du printemps rend pénible à supporter. Mais dans le même temps où ce vide est exhibé, le moyen de le combler s'avance... sous la forme du chevalier Gillebert, chetif sortant, telle Vénus, des ondes (v. 110), hâve et abîmé de son séjour dans les geôles sarrasines. Là encore, une ironie subtile colore le texte et interdit de voir dans ces strophes vernales un simple recours à une tradition formulaire.

Signalons pour finir un chant particulier, celui du coq, annonçant le lever du jour dans le Guillaume et le Girart de Roussillon. Échos de celui qui sonna le reniement de Pierre au matin du jour de l'arrestation de Jésus, le cri du gallinacé ouvre, à chaque fois, une journée fatidique, celle qui va faire tomber la citadelle de Roussillon, par traitrise, aux mains des Royaux (Girart de Roussillon, v. 933 et v. 6278). En outre, le chant du coq est évoqué par des personnages : dans l'esprit des hommes de Guillaume (Chanson de Guillaume, v. 2904) ou dans celui de Fouques (Girart de Roussillon, v. 1610), c'est à une heure fort matutinale qu'il est fait référence, mais le substrat évangélique ne saurait être complètement oblitéré. Plus intéressante est l'occurrence que l'on trouve dans la bouche de la comtesse Berthe à la fin du Girart (v. 9588). Anz quel jals cant est l'expression dont elle se sert pour donner rendez-vous au pèlerin qui a entrepris la construction de Vézelay et qu'elle veut aider. La journée qui suivra ce labeur nocturne ne peut être un jour de colère, bien au contraire, la comtesse, par sa pénitence, ouvre ainsi un temps nouveau dans la geste du révolté, celui de l'apaisement dans la lumière blanche et sacrée de Dieu.

5. Les chevaliers de Constantinople, royaume de paix et de prospérité, se présentent aux Francs portant falcuns et osturs à leur épaule (Voyage de Charlemagne..., v. 271). Faisant croire à une pacifique partie de chasse, Charles et ses hommes pénètrent dans les bois de Roussillon aurions a la fort peine tandis que Girart, qui ne se doute de rien, nourrit le sien (Girart, v. 671 et 728). Mais c'est la fin de ce poème qui est la plus explicite : des querelleurs qu'anc nen amerent paz ne joc d'ostor (v. 9408) refusent d'entendre les paroles de concorde du pape et une fois les tensions définitivement apaisées, Girart lègue à Fouques ses biens terrestres : E serent de sazun chien e ostor, / falcon e falconer $e$ veneor (v. 9974-9975). On verra aussi J.-O. Benoist, " La Chasse au 
vol. Techniques de chasse et valeur symbolique de la volerie », $L a$ Chasse au Moyen Âge. Actes du colloque de Nice, 22 au 22 juin 1979, Paris, Les Belles Lettres, 1980, p. 117-130 et A. Labbé, « Le Château et ses territoires de chasse dans la chanson de geste de Girart de Roussillon ", Le Château, la chasse et la forêt, sous la direction d'A. Chastel, Les Cahiers de Commarque, Bordeaux, 1990, p. 235-249, et l'étude de B. Van den Abeele, La Fauconnerie dans les lettres françaises $d u \times X^{e}$ au XIV ${ }^{e}$ siècle, Louvain, 1990.

6. Le trouvère du Roland insiste sur les mil hosturs müers (v. 31, 129 et 184) que Marsile se propose de donner à Charlemagne ; au moment de solliciter l'aide de ses hommes, Guillaume leur rappelle sa largesse : en temps de paix il ne les a jamais dépouillés de leurs bêtes de chasse et leur a même donné des siennes (Guillaume, v. 1569-1573) ; quant à Girart, il se voit offrir, au début du poème par l'empereur de Constantinople (v. 304), à la fin par Charles en signe de paix (v. 9518), auriuns mudaz et falcon volent.

7. La Chanson de Guillaume, v. 1404-1411.

8. Le Voyage de Charlemagne... Nous reproduisons la transcription diplomatique de l'éditeur, à laquelle nous ajoutons, quand cela s'avère nécessaire pour des questions de métrique, un tréma pour faire apparaître un hiatus et dans laquelle nous distinguons $v$ de $u$.

9. La Prise d'Orange, v. 172-174 et 552-553.

10. Raoul de Cambrai, v. 1382.

11. Girart de Roussillon, v. 6786 et v. 4079.

12. Sur le sujet, voir J.-P. Sosson, " La Part de gibier dans l'alimentation médiévale. L'exemple des " pourvances » de Guillaume d'Ostrevant au Quesnoy (23 sept. 1397-23 juin 1398) ", La Chasse au Moyen Âge, op. cit., p. 347-364 ; J.-R. Trochet, " Chasse et alimentation dans le village médiéval de Dracy », La Chasse au Moyen Âge, op. cit., p. 365-373 ; B. Laurioux, Manger au Moyen Âge, Paris, 2002 (en particulier les p. 68-69 et 72-74).

13. Le Viandier de Taillevent et le Ménagier de Paris étudiés par A. Saly, (« Les Oiseaux dans l'alimentation médiévale d'après le Viandier de Taillevent et le Ménagier de Paris ", Manger et Boire au Moyen Âge. Cuisine, manières de table, régimes alimentaires, Actes du colloque de Nice (15-17 oct. 1982), t. 2, Paris, Belles Lettres, 1984, p. 173-179), offrent pourtant à la fin du Moyen Âge une liste impressionnante par sa longueur et par sa variété de recettes permettant de préparer toutes sortes de volailles et de gibier à plume.

14. M. Montanari, La Faim et l'Abondance. Histoire de l'alimentation en Europe, Paris, Seuil, 1995, en particulier p. 13-96. 
15. On sait l'ambiguïté des images véhiculées par le paon et le cygne, oiseaux qui égarent les sens par la beauté de leur plumage. Le paon, dont la roue imite le soleil et les yeux le ciel étoilé, est aussi le symbole de la vanité. Le cygne, comme le rappelle la Queste del saint Graal (éd. A. Pauphilet, Paris, Champion, CFMA 33, 1984, p. 185-186), blanc de plume, mais noir de peau, représente l'hypocrisie. Toutefois, cette ambivalence n'est pas présente dans les textes qui font l'objet de cette étude.

16. Il ne leur donna pas des mets délicats, comme des pluviers, mais du pain d'orge pétri avec de la cendre et de l'eau de source, froide et douce.

17. Sur l'itinéraire spirituel du héros, nous renvoyons à l'introduction de l'édition qui sert de base à nos citations et à $\mathrm{M}$. de Combarieu du Grès, L'Idéal humain et l'Expérience morale chez les héros des chansons de geste (des origines à 1250), 2 vols, Aix-en-Provence, 1979, en particulier les pages 665-755.

18. B. Laurioux, op. cit., p. 132-135.

19. M. Montanari, op. cit., p. 49-53 et71-76.

20. M. Montanari, op. cit., p. 26-31.

21. Voir J. Grisward, "Paris, Jérusalem, Constantinople dans le Pèlerinage de Charlemagne. Trois villes, trois fonctions ", Jérusalem, Rome, Constantinople. L'image et le mythe de la ville au Moyen Âge, Paris, 1986, p. 75-82.

22. Sur l'importance du poivre dans la cuisine du haut Moyen Âge, on se reportera à B. Laurioux, op. cit., p. 36-37 et 88-89.

23. B. Laurioux, op. cit., p. 132-146.

24. La Chanson de Guillaume, laisses LXXXV et CIII. Pour la mention du temps écoulé, voir la laisse LXXXIX.

25. Voir J.-M. Fritz, « La Théorie humorale comme moyen de penser le monde. Limites et contradictions du système ", Écriture et Modes de pensée au Moyen Âge, Paris, ENS, 1993, p. 13-26. Cette étude n'ayant pas pour but d'établir un diagnostic sur la nature du tempérament des héros épiques (pour une application au domaine romanesque, on pourra consulter l'article de C. Connochie-Bourgne, "Lancelot et le tempérament colérique », De la Science en littérature à la sciencefiction, actes du CXIX ${ }^{e}$ congrès des sociétés historiques et scientifiques (Amiens, 24-28 oct. 1994), CTHS, Paris, 1996, p. 11-22), nous nous contentons d'une approximation par l'âge.

26. La Chanson de Guillaume, laisses CLVII et CLVIII.

27. C. Casagrande et S. Vecchio, Les Péchés de la langue. Discipline et 
éthique de la parole dans la culture médiévale, Paris, Cerf, 1991 pour la traduction française. Le chapitre V, p. 113-135, lie explicitement gourmandise et péché de langue. Des mêmes, Histoire des péchés capitaux au Moyen Âge, Paris, Aubier, 2003 pour la traduction française, en particulier les pages 193-228.

28. Le Couronnement de Louis, éd. E. Langlois, Paris, Champion, 1984, v. 2213, 2253, 2674.

29. Voir R. Klibansky, E. Panofsky et F. Saxl, Saturne et la Mélancholie, p. $180, \mathrm{n}^{\circ} 137$.

30. La faim et la soif endurées sur le champ de bataille sont un leitmotiv qui parcourt tout le poème, venant en rajouter sur la souffrance des hommes. On pourra consulter l'étude de M. de Combarieu du Grès, "Bonnes et mauvaises manières de table dans $L a$ Chanson de Guillaume et Aliscans ", Banquets et Manières de table au Moyen Âge, Senefiance 38, Aix-en-Provence, 1996, p. 282-301.

31. Enfin, il est peut-être permis de penser que la dénonciation de Guillaume en colère va encore plus loin. C'est, en effet, le quantifiant possessif tes qui détermine pudcins, établissant un lien étroit d'appartenance entre la reine et ce qu'elle mange en même temps que son pouvoir sur ces animaux. Anthony Rowley, Une Histoire mondiale de la table. Stratégies de bouche, Paris, O. Jacob, 2006, lit dans le goût pour les petits oiseaux, les restes d'un vieux mythe de fécondation masculine : " Le goût des Méditerranéens pour les petits oiseaux synthétise par exemple l'héritage probable des mythes de la fécondation masculine, à la fois dans la forme ronde et petite d'animaux rapprochables des testicules, dans le mode de cuisson où la chaleur extrême du plat fait penser à la " cuisson complète » de la copulation, dans l'ingestion entière de l'animal qui rapproche un moment du cannibale - un archétype de la virilité - et enfin dans la rareté de l'oiseau qui en fait une parure remarquable pour un repas. » p. 60. Une fois encore ressurgit, à propos de la reine, le spectre de la nature dévoyée, voire niée : une femme ingérant un organe de reproduction masculin, extrême orgueil de celle qui, dans un acte de déglutition, prétend anéantir le sexe fort !

32. Raoul de Cambrai, v. 323 et 1094.

33. À propos de l'épisode d'Origny du Raoul, on consultera, entre autres, M. de Combarieu du Grès, Âge cit., t. 2, p. 645-655, L'Orgueil a desmesure. Études sur Raoul de Cambrai, Caen, Paradigme, 1999 (en particulier A. Labbé, " La Croix, l'épée et la flamme. Autour de l'incendie d'Origny dans Raoul de Cambrai », p. 147-185), Raoul de Cambrai, ouvrage dirigé par J.-Cl. Vallecalle, Paris, Ellipses, 1999 (en particulier, B. Guidot, "Spiritualité et violence dans Raoul de 
Cambrai », p. 21-37 et A. Labbé, " Défi et blasphème dans l'épisode d'Origny de Raoul de Cambrai : impiété ou désir de Dieu ? », p. 57-66).

34. Voir les mots de Guerri v. 1098-1106 et ceux du sénéchal v. 13891398.

35. Le ms $F$ donne au v. 5806 : plain de ploviers, qui chaut sont et rosti.

36. L'épisode est plus rapide dans $F$ (v. 5807-5826) : Begon brisant son arme après le coup porté à Ysoré, il se contente, avec le tronçon restant d'abattre Hardouin. On ne trouve qu'une seule hypotypose v. 58125813 .

37. On songera que les choses vont encore empirer puisque les retrouvailles entre les deux frères lorrains, après l'épisode du siège de Bordeaux consécutif à leurs mariages respectifs, ne se feront pas : c'est la dépouille de Bégues que l'on amènera à Garin.

38. Sur la lecture dumézilienne du couple fraternel lorrain, on consultera les articles de J. Grisward, « Le Thème de la révolte dans les chansons de geste : éléments pour une typologie du héros révolté ", Charlemagne in the North. proceedings of the Twelfth International Conference of the Société Rencesvals, (Edinbourg, 4-11 août 1991), Edinbourg, 1993, p. 399-416 et « La Cuisineet la guerre : aspects de la fonction guerrière dans la Geste des Lorrains ", L'Épopée romane. Actes du XV $\mathrm{XV}^{\mathrm{e}}$ congrès international Rencesvals, (Poitiers, 21-27 août 2000), Poitiers, 2002, p. 549-565. Plus largement sur les relations qui unissent les deux frères, on verra $\mathrm{M}$. de Combarieu du Grès, "Les "Amis charnels" (Garin et Bègue dans Garin le Lorrain)", Histoire et Société : le couple, l'ami et le prochain. Mélanges offerts à G. Duby, vol. I, Aix-en-Provence, 1992, p. 125-139.

\section{Auteur}

\section{Valérie Naudet}

\section{Université de Provence - Aix- Marseille I}

(C) Presses universitaires de Provence, 2009

Conditions d'utilisation : http://www.openedition.org/6540 
NAUDET, Valérie. Des poussins au poivre, volailles aux tables épiques In : Déduits d'oiseaux au Moyen Âge [en ligne]. Aix-en-Provence : Presses universitaires de Provence, 2009 (généré le 27 avril 2017). Disponible sur Internet : <http://books.openedition.org/pup/4290>. ISBN : 9782821836877 . DOI : 10.400o/books.pup.4290.

\section{Référence électronique du livre}

CONNOCHIE-BOURGNE, Chantal (dir.). Déduits d'oiseaux au Moyen Âge. Nouvelle édition [en ligne]. Aix-en-Provence : Presses universitaires de Provence, 2009 (généré le 27 avril 2017). Disponible sur Internet : <http://books.openedition.org/pup/4252>. ISBN : 9782821836877. DOI : 10.400o/books.pup.4252.

Compatible avec Zotero 